\title{
Multiplexed End-point Microfluidic Chemotaxis Assay using Centrifugal Alignment
}

Sampath Satti, a,b Pan Deng, ,,c Kerryn Matthews, ${ }^{\text {b,c }}$ Simon P. Duffy, ${ }^{\text {b,c,d }}$ and Hongshen Ma*a,b,c,e

\section{Affiliations:}

${ }^{a}$ School of Biomedical Engineering, University of British Columbia

${ }^{\mathrm{b}}$ Centre for Blood Research, University of British Columbia

${ }^{\mathrm{c}}$ Department of Mechanical Engineering, University of British Columbia

${ }^{\mathrm{d}}$ British Columbia Institute of Technology

${ }^{\mathrm{e}}$ Department of Urologic Sciences, University of British Columbia

* Correspondence should be addressed to Hongshen Ma (hongma@mech.ubc.ca)

Keywords: Chemotaxis, Microfluidics, Cell Alignment, Centrifugation

\begin{abstract}
A fundamental challenge to multiplexing microfluidic chemotaxis assays at scale is the requirement for time-lapse imaging to continuously track migrating cells. Drug testing and drug screening applications require the ability to perform hundreds of experiments in parallel, which is not feasible for assays that require continuous imaging. To address this limitation, end-point chemotaxis assays have been developed using fluid flow to align cells in traps or sieves prior to cell migration. However, these methods require precisely controlled fluid flow to transport cells to the correct location without undesirable mechanical stress, which introduce significant set up time and design complexity. Here, we describe a microfluidic device that eliminates the need for precise flow control by using centrifugation to align cells at a common starting point. A chemoattractant gradient is then formed using passive diffusion prior to chemotaxis in an incubated environment. This approach provides a simple and scalable approach to multiplexed chemotaxis assays. Centrifugal alignment is also insensitive to cell geometry, enabling this approach to be compatible with primary cell samples that are often heterogeneous. We demonstrate the capability of this approach by assessing chemotaxis of primary neutrophils in response to an fMLP (N-formyl-metleu-phe) gradient. Our results show that cell alignment by centrifugation offers a potential avenue to develop scalable end-point multiplexed microfluidic chemotaxis assays.
\end{abstract}




\section{Introduction}

Cells have a sophisticated ability to sense gradients of chemoattractants and then respond by directed migration along these gradients via chemotaxis. Chemotaxis underpins a diverse range of biological processes, including infection ${ }^{1}$, wound healing ${ }^{2}$, inflammation ${ }^{3}$, embryogenesis ${ }^{4}$, and cancer metastasis ${ }^{5}$. Consequently, there has been a longstanding interest to develop assays for chemotaxis. Conventional chemotaxis assays are often qualitative and low-throughput. Overcoming these limitations is key to integrating chemotaxis into platforms for diagnosis ${ }^{6}$, drug testing ${ }^{7,8}$ and drug discovery 9 . The traditional approach for evaluating chemotaxis uses the Boyden assay, also known as the Transwell assay, which generate chemical gradients across a porous membrane ${ }^{10}$. The cells that migrate across the membrane are then enumerated to produce a measure of chemotactic capability. While this approach is simple to perform, it often produces inconsistent results because the chemical gradient experienced by each cell across the membrane interface can vary with time, location, and cell density ${ }^{9}$. Transwell assays also require a large number of cells $\left(10^{5}-10^{6}\right)$ and large volumes of chemoattractant solutions, limiting the number and types of experiments that could be performed, especially on primary cell samples obtained from patients ${ }^{11}$.

Microfluidic approaches have been used to develop improved chemotaxis assays that provide more reliable chemical gradients, as well as that reduce the requirements for cells and reagents ${ }^{12-20}$. These devices typically involve generating chemoattractant gradients through passive diffusion or through active perfusion. Cells are then introduced into this gradient in order to observe their migration via time-lapse microscopy. This approach has a limited capacity to perform multiple chemotaxis assays in parallel because of the need to continuously track cell migration in each assay using microscopy. While multiplexing these experiments could be 
61 achieved using automated microscopy, this approach is not scalable for high throughput applications such as drug testing and drug screening ${ }^{21}$. limitation in the scalability of both trap- and sieve-based cell alignment is the requirement for

71 precisely controlled fluid flow in each device to seed cells in the right locations. The flow rate

72 must be matched with the cell type and size in order to align the cells without squeezing past the

73 trap or sieve, or apply shear stress that may affect chemotactic behavior ${ }^{26}$. Consequently, this

74 approach requires lengthy set up time for cell alignment and introduces design constraints that

75 limit the scalability of the assay.

Here, we developed a scalable end-point chemotaxis assay that uses centrifugal force to

77 align cell samples to a common starting point in order to provide a multiplexed end-point chemotaxis assay without the need for continuous microscopy. Individual microfluidic devices are

79 arrayed in a rotational symmetric manner on a glass slide substrate. Unlike trap- or sieve-based alignment, this approach obviates the need for carefully controlled fluid flow by aligning cells via

81 centrifugation against a barrier feature. We validated this approach by assessing the migration of primary neutrophils along an fMLP gradient in order to demonstrate its potential as a simple, scalable, and multiplexed chemotaxis assay requiring minimal equipment. 
84

85

86

87

88

89

90

91

92

93

94

95

96

97

98

100

101

102

103

104

105

\section{Results}

\section{Microfluidic Device Design and Assay Preparation}

The microfluidic device for end-point chemotaxis assays comprise of two reservoirs connected by a thin microchannel that limits diffusion between the reservoirs (Figure 1A \& 1C).

The microfluidic channel contains a barrier feature that enables centrifugal alignment of the cell sample at a common starting point before starting each chemotaxis assay (Figure 1B). The barrier feature includes a small degree of concavity designed to trap cells against movement created by tangential forces (perpendicular to channel) resulting from angular acceleration caused by starting and stopping the spinning process. The microfluidic channel and alignment barrier are formed using PDMS bonded to a glass substrate, which can be functionalized with extracellular matrix (ECM) to enhance chemotaxis. To multiplex chemotaxis experiments, individual devices are arrayed in a rotationally symmetric fashion on a single glass substrate allowing for multiple experiments to be performed simultaneously (Figure 1D). As a demonstration of this approach, we developed a prototype multiplexing 12 separate devices on a single 50 x $75 \mathrm{~mm}$ glass slide substrate.

The multiplexed end-point chemotaxis assay begins by functionalizing the glass surfaces in each microfluidic device using fibronectin, an ECM protein that enhances neutrophil migration in vitro ${ }^{27}$. The devices are then blocked using bovine serum albumin (BSA) to prevent non-specific adhesion. After functionalizing the surfaces, the cell samples are introduced into each device by standard pipette. Next, the microfluidic device is spun in order to align cells in the device against the barrier feature by centrifugation. Cells not aligned against the barrier feature are transported to the reservoir past the barrier feature and generally excluded from the assay. In order to prevent 
106 fluid leakage during the alignment process, the outlets were sealed using acrylic tape. After

107 pipetting the cell sample, the chemoattractant was pipetted into the source reservoir.

108 Chemoattractant molecules were diffused from the source reservoir into the sink reservoir through

109 the gradient channel to establish a gradient (Figure 1B). The device was then placed inside an

110 incubator to allow chemotaxis to take place at stable temperature and gas conditions. Finally, after

111 an appropriate amount of time, the device is imaged to determine the resulting cell positions.

\section{Gradient Formation} without the barrier feature (Figure 2D). The correlation coefficient between gradient strengths in 
129 The Rhodamine B gradient is similar in profile to the FITC-Dextra gradient with a scale factor of 1.59 and a correlation coefficient of 0.99 (Figure S1). This scale factor error can be accounted for by modulating the chemoattractant concentration at the source reservoir.

132 becomes:

\section{Cell Alignment Through Centrifugation}

To align cells against the barrier feature, the microfluidic device is rotated using a spinner to produce a centrifugal force $\left(F_{c}\right)$ towards the center of rotation:

$$
F_{c}=\left(\rho_{n}-\rho_{0}\right) V \omega^{2} l
$$

where $\rho_{n}$ is the density of neutrophils ${ }^{28}$ and $\rho_{0}$ is the density of water, $V$ is the volume of a neutrophil, $\omega$ is the angular velocity, $l$ is the distance from the axis of rotation. For small particles moving in a liquid at low Reynolds number, they need to overcome the Stokes drag force $\left(F_{d}\right)$ :

$$
F_{d}=6 \pi \mu r v
$$

where $\mu$ is the dynamic viscosity of the fluid, $r$ is the radius of small particles or cells, $v$ is the speed of small particles or cells. Since the bottom surface of the microchannel is treated with fibronectin, $F_{c}$ need be sufficiently large to first allow cells to overcome fibronectin adhesion before transporting cells against viscous forces towards barriers. Once cells begin to move, this adhesion force becomes negligible. Additionally, since the length the cells need to move across

the microchannel is much smaller than $l$, the value of $F_{c}$ could be considered constant during transport by centrifugation. When $F_{c}$ is balanced by $F_{d}$, the centrifugal transport velocity $\left(V_{c}\right)$ 
149 If cells could migrate consistently at $V_{c}$ and cells were evenly distributed in channels at the beginning, the number of collected cells at barriers $(N)$ could be calculated by:

$$
N=W V_{c} t \rho_{d}=k \omega^{2}
$$

152 Where $W$ is the width of migration channel, $t$ is the centrifugal spinning time, $\rho_{d}$ is the cell distribution density, $k$ is a constant.

To experimentally confirm our model, we loaded the microfluidic device with human neutrophils $\left(1.4 \times 10^{6} / \mathrm{ml}\right)$ and centrifuged the device at speeds from 0 to $2000 \mathrm{RPM}$ for one minute and then counted the number of collected cells $(n=5$, Figure $3 \mathbf{A}-\mathbf{D})$. We found the number of collected cells to monotonically increase from 0 to 1500 RPM. At 2000 RPM, however, the number of collected cells decreased due to excessive tangential forces arising from spinner acceleration and deceleration. This issue could be resolve in future versions using a larger concavity. We then measured the value of $k$ by fitting Equation (4) to data points from 0 to 1500 RPM and found a high-quality fit $\left(R^{2}>0.98\right.$, Figure 3E). Based on these results, we selected 1500 RPM for $1 \mathrm{~min}$ as the condition for cell alignment throughout our study.

\section{Chemotaxis Validation}

To validate neutrophil chemotaxis in the microfluidic device, we performed chemotaxis assay in gradients of fMLP using human neutrophils. Neutrophils were isolated from whole blood

166 using a commercial magnetic immunoselection kit and resuspended in a cell migration media.

167 Cells were infused into the device after fibronectin coating and BSA blocking. After a short 168 incubation period, a chemoattractant gradient was generated by pipetting fMLP solution into the 169 source reservoir. Time lapse images of neutrophil migration were recorded on an inverted 170 microscope and cell tracking performed through an ImageJ plugin (Video S2). Over 85\% of cells 
171 in fMLP gradients had an elongated morphology in the first few minutes when compared to the

172 rounded morphologies seen in the absence of fMLP gradient in control devices. Analyzing the cell

173 tracks at an individual cell level revealed minimal migration in control devices (Figure 4A), but

174 substantial directional bias of neutrophil migration in $100 \mathrm{nM}$ fMLP gradients, with $80 \%$ of all

175 cells moving towards the source of the chemoattractant (Figure 4B). In the $100 \mathrm{nM}$ fMLP gradient,

176 neutrophils had an average migration velocity of $2 \mu \mathrm{m} / \mathrm{min}$ and the velocity did not significantly

177 change after 30 minutes (Figure S2). The cell paths were found to have an average directness

178 factor of 0.3 (ratio of displacement to total distance), showing a strong preference for fMLP

179 gradient. In contrast, the control sample showed no net displacement and did not possess a

180 directional bias. We further assessed the variation of neutrophil migration speed as a function of

181 time, which stabilized after $\sim 30 \mathrm{~min}$ as the gradient reached stable values (Figure S2). These

182 results confirm that our microfluidic device is able to elicit a chemotactic response from the added

183 cell sample.

\section{End-point Analysis Validation}

To establish the effectiveness of the end-point chemotaxis assay, we repeated the neutrophil chemotaxis experiments with the additional step of centrifugal alignment. We determined the locations of cells, relative to the alignment barrier, by automatic segmentation of a microscope image of each channel after two hours of incubation at $37^{\circ} \mathrm{C}$. The end-point chemotaxis assay was validated by comparing the final positions of neutrophils after incubation in devices with chemoattractant gradients created using various fMLP concentrations. In control

191 devices without fMLP gradient, only a few cells were observed to migrate away from the barrier.

192 In devices with fMLP gradient, most of the cells were observed to migrate away from the barrier

193 (Figure 5A-C). Varying the fMLP gradient, we found that neutrophils appear to require a 
threshold fMLP gradient to activate chemotaxis since migration was observed only when the fMLP gradient was greater or equal to $25 \mathrm{nM}$ (Figure 5D-F, p $<0.005)$. There also appears to be a decrease in migration distance from $25 \mathrm{nM}$ to $100 \mathrm{nM}$, but this trend was not statistically significant $(\mathrm{p}>0.05)$. Together, these data present support the use of centrifugal cell alignment as a rapid and convenient multiplexed end-point cell migration assay.

\section{Discussion}

In this study, we investigated an approach to develop a multiplexed end-point chemotaxis assay by aligning the cell sample against a barrier via centrifugation. The aligned cells are then exposed to a passively generated chemoattractant gradient, which causes the cells to migrate away from the barrier. Fluorescence imaging was used to observe chemical gradient profiles and validate its stability up to 3 hours while cell alignment at the barrier feature after centrifugation at various spin speeds was quantified through microscopy. Neutrophils in gradients of fMLP showed elongated morphologies and directed migration, while cells in control devices were rounded and non-motile. End-point analysis was used to obtain the average displacement of the cell population in response to the gradient as well as the spread in the final positions of cells after migration. After migration in an incubated environment, the chemotactic capability of the cell sample could be determined from a single microscopy image of the cells in the device.

Recent work on microfluidic chemotaxis assays often require real-time microscopy to track cell migration paths. While these experiments can be multiplexed using automated image acquisition, the requirement for cell tracking places fundamental limits on the throughput of these assays. Specifically, tracking fast moving cells, such as neutrophils, require acquiring an image every 30 seconds. The time required for moving the microscope stage and for image acquisition places a limit on the number of assays that can be analyzed in parallel. Experimental throughput 
217 can be increased by using advanced cell tracking algorithms that do not assign individual cell

218 tracks, but instead derives aggregated populational measurements from the sample ${ }^{21}$. However,

219 this approach does not fundamentally improve the scalability of these assays.

End-point chemotaxis assays that align the cell sample at a common starting point can

221 dramatically improve the throughput and scalability of chemotaxis assays by providing a readout

222 from a single microscopy image. Previous efforts to develop end-point chemotaxis assays used

223 traps and sieves to position cells at a common starting point. The cells must be transported into

224 these locations using carefully controlled fluid flow, which involves long preparation times and

225 can require large volumes of reagents, and is therefore difficult to scale. Aligning cells using traps

226 and sieves also applies significant shear stress on the cells, which can affect their migratory

227 properties ${ }^{26}$. Additionally, the geometry of the traps or sieve must be matched to the geometry of

228 the cell as well as the density of the cell sample, which makes this approach less robust when

229 dealing with heterogeneous samples, such as primary samples from patients. Our centrifugal

230 alignment method provides an alternative approach for cell alignment that does not require precise

231 flow control, applies minimal shear stress to cells, and is largely insensitive to cell geometry. Cells

232 can simply be pipetted into the device, where they are centrifuged to align against a concave barrier

233 feature designed to minimize inertial shear. The motion of cells within the device is primarily

234 driven by centrifugation speed, which can be easily adjusted based on the cell sample, device

235 geometry, and surface adhesion forces. This approach is also compatible with heterogeneous cell

236 samples where target cells can be fluorescently labeled and then identified by imaging after

237 chemotaxis. Therefore, centrifugation-based alignment is an attractive option for developing

238 scalable multiplexed end-point chemotaxis assays. 
Finally, the microfluidic device design described here provides a number of favourable

240 features that enable high-throughput experiments by scaling to hundreds of simultaneous

241 chemotaxis assays in parallel. Specifically, the microfluidic device has a small footprint, which

242 enables the integration of a large number of experiments on a single substrate. The design is a

243 simple single-layer device that is easy to manufacture and does not require precise mechanical

244 assembly. The device preparation and cell alignment does not require external controls for fluid

245 handling thus leading to simple operation that can be automated using a pipetting robot. Each

246 individual device requires only a small number of cells $(500-5000$ cells $/ \mathrm{ml})$ and reagents $(5 \mu 1)$.

247 Centrifugation allows for simultaneous alignment of a wide range of cell types seeded at various

248 densities. All these features result in a stand-alone multiplexed device that can be infused with

249 reagents and cell samples and then simply be placed in the incubator for chemotactic migration. In

250 conclusion, we demonstrate a simple and scalable multiplexed end-point chemotaxis where cells

251 are aligned to a common starting point by centrifugation.

252

253

254 


\section{Materials and Methods}

\section{Preparation of Primary Neutrophils}

Healthy donors between the ages of 18 and 70 were included in this study. Following

Neutrophils were washed and resuspended in RPMI-1640 (Cat \#11875119, Gibco) with 0.4\% low speed. All purified neutrophils were used within 2 hours after the separation.

\section{Device Fabrication}

Our microfliudic device consists of 12 microchannels with two reservoirs (diameter $3 \mathrm{~mm}$, height $2 \mathrm{~mm}$ ) connected by a straight channel (length $2 \mathrm{~mm}$, width $1 \mathrm{~mm}$, height $0.3 \mathrm{~mm}$ ). The

271 alignment feature/barrier is $0.4 \mathrm{~mm}$ long, $0.3 \mathrm{~mm}$ wide and with a concavity on one edge. 3D

272 printed molds of the device (Protolabs, Raleigh, USA) were used for device fabrication. The

273 moulds were pre-treated at $65^{\circ} \mathrm{C}$ for 48 hours prior to use. PDMS silicone (Sylgard-184, Ellsworth

274 Adhesives, Germantown, WI, USA) was mixed at a 10:1 ratio with the curing agent (Sylgard-184,

275 Ellsworth Adhesives, Germantown, WI, USA) and poured into the molds. The molds were 276 degassed and heat-cured for 2 hours at $65^{\circ} \mathrm{C}$. The cured PDMS was removed from the molds and 277 inlet holes for the source and sink reservoirs were punched using a $1 \mathrm{~mm}$ hole punch (Technical 
Innovations, Angleton, TX, USA). The device was then bonded to a 2" x 3" glass slide (TedPella Inc, USA) using air plasma (Cat \#PDC-001, Harrick Plasma). The freshly bonded device was immediately filled with phosphate-buffered saline (Cat \#10010023, Gibco) to prevent air bubble formation.

\section{Device Preparation and Cell Alignment}

The plasma activated device was infused with $30 \mu \mathrm{l}$ fibronectin $(0.1 \mathrm{mg} / \mathrm{ml})$ extracellular matrix (ECM) protein solution in PBS and incubated one hour before flushing with $200 \mu \mathrm{L}$ of Phosphate Buffered Saline. Subsequently, the device was primed by infusion of $30 \mu 10.4 \%$ BSA/RPMI, followed by incubation for one hour at room temperature. The device was then flushed with $30 \mu 1$ fresh $0.4 \%$ BSA/RPMI, followed by seeding of $15 \mu 1$ enriched neutrophils at the desired cell density. To align cells within the device, the inlets were then sealed with tape (Cat \#96042, 3M) and the device was transferred to a Headway spinner at 2000 RPM for one minute, followed by incubation at $37^{\circ} \mathrm{C}$ for 15 minutes.

\section{Gradient Formation and Chemotaxis Assay}

A chemoattractant solution was made by preparing $100 \mathrm{nM}$ N-Formyl-Met-Leu-Phe (fMLP, Millipore Sigma) in $0.4 \%$ BSA/RPMI, supplemented, with $0.1 \% \mathrm{w} / \mathrm{v}$ FITC-dextran (Millipore Sigma). Immediately following cell alignment, $5 \mu \mathrm{L}$ this fMLP solution was introduced into the source reservoir and a thin layer of silicone oil (Cat \#378399, MilliporeSigma) was applied to cover the openings to minimize evaporation. For continuous cell tracking, the device was imaged with a Nikon Ti-2 microscope. The images were analyzed using the FIJI cell tracking plugin from Image ${ }^{29}$. The cell paths obtained were later parsed and analyzed using custom Python scripts to visualize raw data. For end-point analysis, the microfluidic device was incubated in a 
$37^{\circ} \mathrm{C}$ humidified incubator for two hours. The device was subsequently imaged and cell migration distance was measured using ImageJ.

\section{Simulation of Gradient Formation}

COMSOL Multiphysics was used to simulate and validate the gradient progression and evolution in the device. The simulation was established based on device geometry imported directly from the CAD file used for fabrication. The laminar flow profile and transport of diluted species physics modules were used to model the gradient of FITC Dextran $\left(\mathrm{D}=6 \times 10^{-6} \mathrm{~cm}^{2} \mathrm{~s}^{-1}\right)$. No slip conditions were applied to obtain the initial profile of the gradient due to flow. The simulation assumed chemoattractant solution was introduced into the device at a flow rate of $1 \mu 1 / \mathrm{s}$ for 5 seconds.

\section{Additional Information}

Conflicts of Interest: There are no conflicts of interests to declare.

Acknowledgments: This work was supported by grants from a NSERC (2015-06541, 508392-

17) and CIHR (381129). P.D. acknowledges funding from the China Scholarship Council. K.M. acknowledges funding from MITACS Accelerate program (IT09621). H.M. acknowledges funding from the CIHR New Investigator Salary Award program (322375).

Authorship Contributions: H.M. conceived the idea and supervised the work. S.S., P.D., and K.M. performed the experimental work. All authors wrote the manuscript. 


\section{References}

325 1. Smith, P. D. et al. Monocyte function in the acquired immune deficiency syndrome. Defective chemotaxis. The Journal of clinical investigation 74, 2121-8 (1984).

2. de Oliveira, S., Rosowski, E. E. \& Huttenlocher, A. Neutrophil migration in infection and wound repair: going forward in reverse. Nature Reviews Immunology 16, 378-391 (2016).

3. Phillipson, M. \& Kubes, P. The neutrophil in vascular inflammation. Nature Medicine 17, 1381-1390 (2011).

4. Dormann, D. \& Weijer, C. J. Chemotactic cell movement during development. Current Opinion in Genetics \& Development 13, 358-364 (2003).

5. Roussos, E. T., Condeelis, J. S. \& Patsialou, A. Chemotaxis in cancer. Nature reviews. Cancer 11, 57387 (2011).

6. Sackmann, E. K.-H. et al. Characterizing asthma from a drop of blood using neutrophil chemotaxis. Proceedings of the National Academy of Sciences of the United States of America 111, 5813-8 (2014).

7. Lakshman, M. et al. Dietary genistein inhibits metastasis of human prostate cancer in mice. Cancer Research 68, 2024-2032 (2008).

8. Sapey, E. et al. Simvastatin Improves Neutrophil Function and Clinical Outcomes in Pneumonia: a Pilot Randomised Controlled Trial. American Journal of Respiratory and Critical Care Medicine rccm.201812-23280C (2019) doi:10.1164/rccm.201812-23280C. leucocytes. The Journal of Experimental Medicine 115, 453 (1962). 
11. Guidelines for Use: Transwell ${ }^{\circledR}$ Permeable Supports | Life Sciences | Corning.

https://www.corning.com/worldwide/en/products/life-sciences/products/permeablesupports/transwell-guidelines.html.

12. Jeon, N. L., Baskaran, H., Dertinger, S. K. W. \& Whitesides, G. M. Neutrophil chemotaxis in linear and complex gradients of interleukin-8 formed in a microfabricated device. 20, (2002).

13. Boneschansker, L., Yan, J., Wong, E., Briscoe, D. M. \& Irimia, D. Microfluidic platform for the quantitative analysis of leukocyte migration signatures. Nature Communications 5, 4787 (2014).

14. Abhyankar, V. V, Lokuta, M. a, Huttenlocher, A. \& Beebe, D. J. Characterization of a membranebased gradient generator for use in cell-signaling studies. Lab on a chip 6, 389-93 (2006).

15. Walsh, D. I., Lalli, M. L., Kassas, J. M., Asthagiri, A. R. \& Murthy, S. K. Cell chemotaxis on paper for diagnostics. Anal. Chem. 87, 5505-5510 (2015).

16. Song, J. et al. A microfluidic device for studying chemotaxis mechanism of bacterial cancer targeting. Sci Rep 8, 6394 (2018).

17. Mehling, M., Frank, T., Albayrak, C. \& Tay, S. Real-time tracking, retrieval and gene expression analysis of migrating human T cells. Lab Chip 15, 1276-1283 (2015).

18. Shen, C. et al. Bacterial chemotaxis on SlipChip. Lab Chip 14, 3074-3080 (2014).

19. Cho, H., Hamza, B., Wong, E. A. \& Irimia, D. On-demand, competing gradient arrays for neutrophil chemotaxis. Lab Chip 14, 972-978 (2014).

20. Sackmann, E. K. et al. Microfluidic kit-on-a-lid: a versatile platform for neutrophil chemotaxis assays. Blood 120, e45-e53 (2012).

21. Berthier, E., Surfus, J., Verbsky, J., Huttenlocher, A. \& Beebe, D. An arrayed high-content chemotaxis assay for patient diagnosis. Integr Biol (Camb) 2, 630-638 (2010).

22. Yang, K. et al. A dual-docking microfluidic cell migration assay (D2-Chip) for testing neutrophil chemotaxis and the memory effect. Integrative Biology (United Kingdom) 9, 303-312 (2017). 
371 23. Poudineh, M. et al. Profiling Functional and Biochemical Phenotypes of Circulating Tumor Cells

372 Using a Two-Dimensional Sorting Device. Angewandte Chemie - International Edition 56, 163-168

373 (2017).

374 24. Chen, Y.-C. et al. Single-cell Migration Chip for Chemotaxis-based Microfluidic Selection of

$375 \quad$ Heterogeneous Cell Populations. Scientific reports 5, 9980 (2015).

376 25. Wu, J., Kumar-Kanojia, A., Hombach-Klonisch, S., Klonisch, T. \& Lin, F. A radial microfluidic platform

377 for higher throughput chemotaxis studies with individual gradient control. Lab Chip 18, 3855-3864

$378 \quad$ (2018).

379 26. Walker, G. M. et al. Effects of flow and diffusion on chemotaxis studies in a microfabricated gradient 380 generator. Lab Chip 5, 611-618 (2005).

381 27. Everitt, E. A., Malik, A. B. \& Hendey, B. Fibronectin enhances the migration rate of human neutrophils in vitro. J. Leukoc. Biol. 60, 199-206 (1996). and in patients with acute lymphoblastic leukemia. Blood 48, 361-371 (1976). 36-42 (2004). 


\section{Figures}
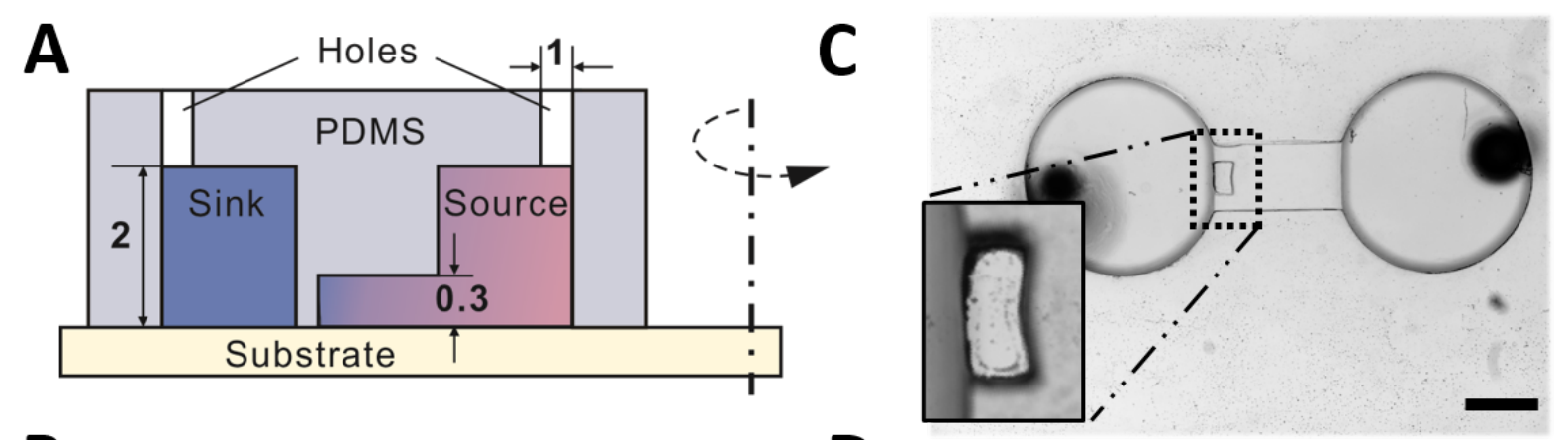

B

D
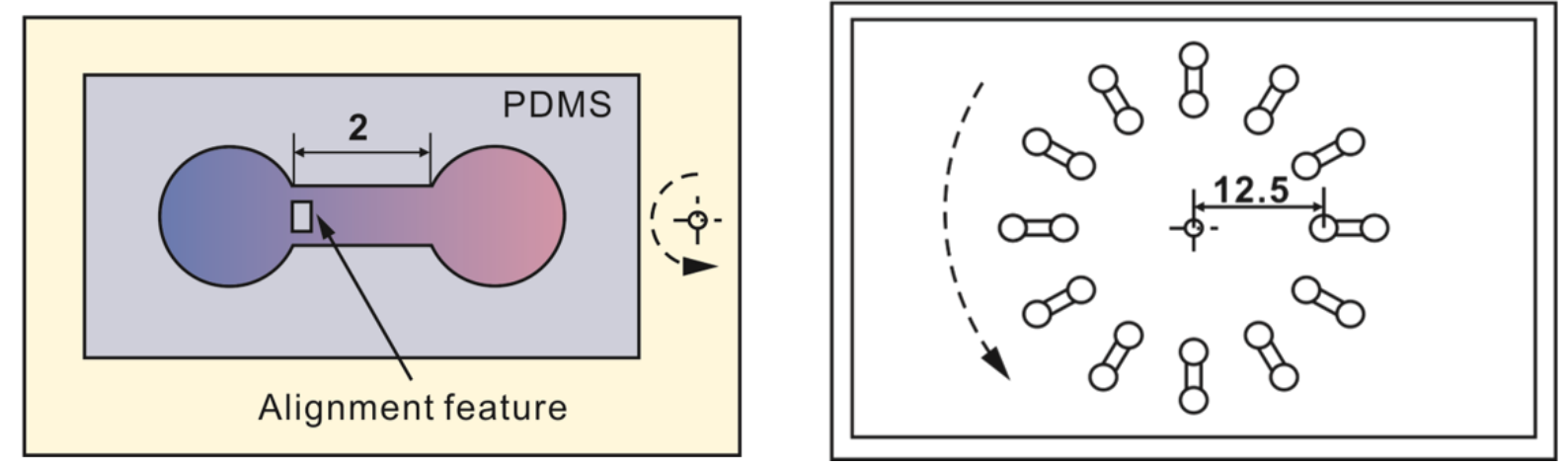

Figure 1. Design of the multiplexed end-point chemotaxis assay. (A-B) Cross-section and topview schematics of the microfluidic device design for an individual chemotaxis assay, including the alignment barrier and critical dimensions (in $\mathrm{mm}$ ). Chemoattractant gradients are generated by passive diffusion between the source and sink reservoirs. (C) Micrograph of an individual chemotaxis assay. Scale bar $=1000 \mu \mathrm{m}$. Inset: Concave barrier for cell alignment. (D) Design of the 12-plex end-point chemotaxis assay where cell alignment is achieved through centrifugal alignment. Radial position of the alignment feature in $\mathrm{mm}$. 

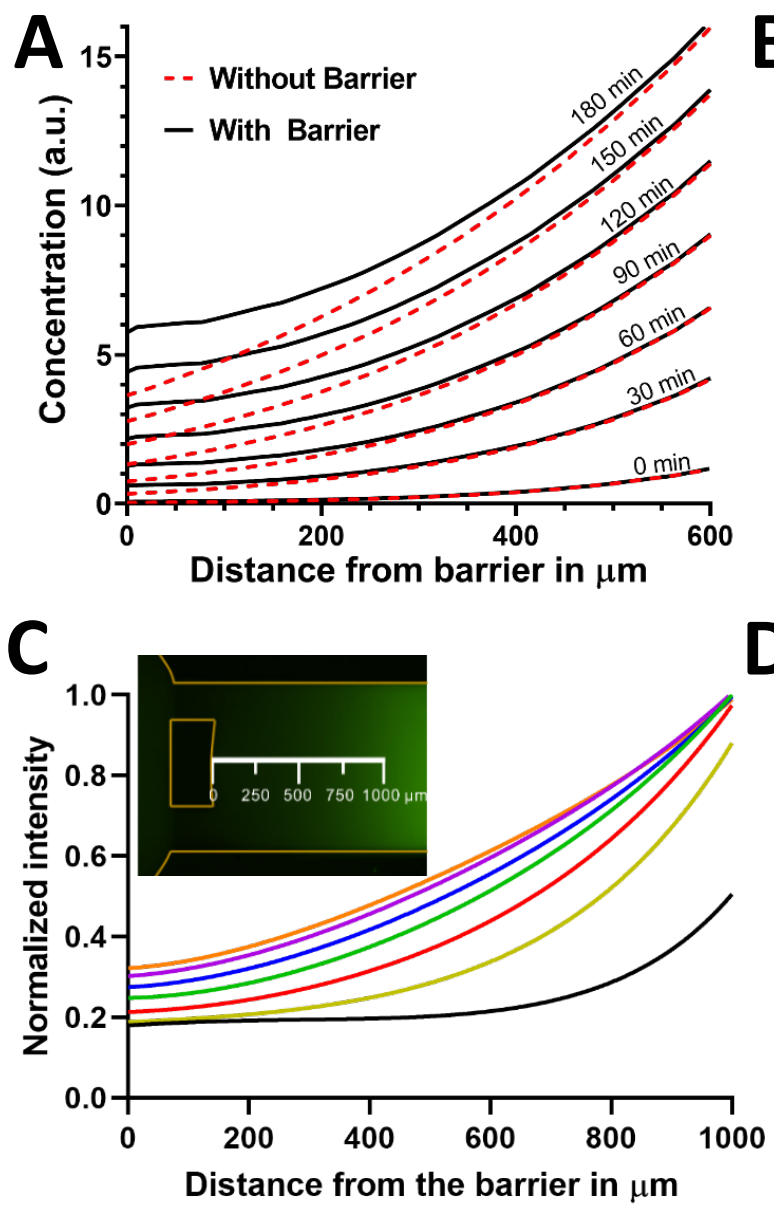

B

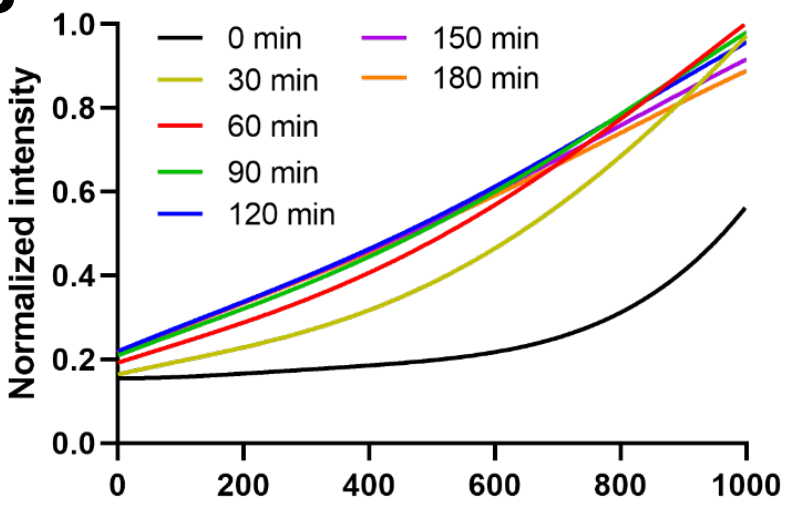

Distance from the source reservoir in $\mu \mathrm{m}$

D

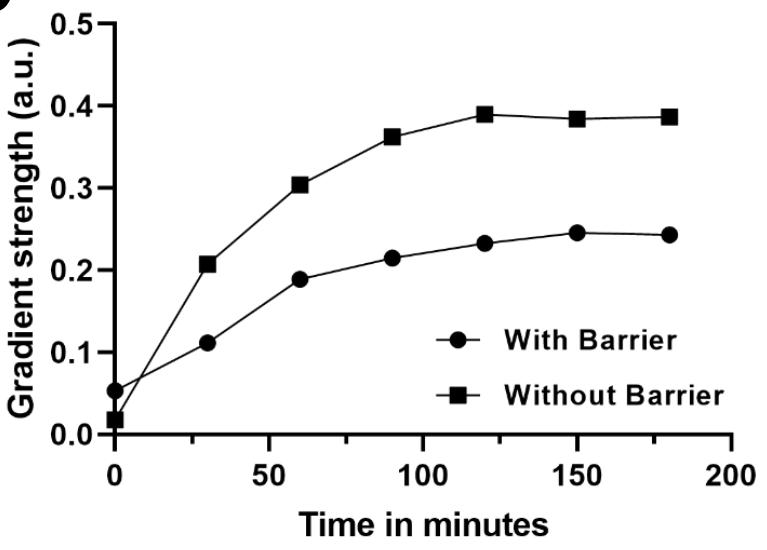

Figure 2. Simulation and experimental study of gradient generation using passive diffusion.

(A) Finite element simulation of the gradient profile with and without the alignment barrier from 0 to 180 minutes. (B-C) Experimental study of gradient profiles without the barrier feature (B) and with the barrier feature $(\mathrm{C})$. The gradient profile was visualized using fluorescent FITC-Dextran (inset in C). (D) The gradient strength measured at $\mathrm{x}=0 \mu \mathrm{m}$ (defined in inset in C) as a function of time for microfluidic devices with and without the barrier feature (correlation coefficient $>0.96$ ). 

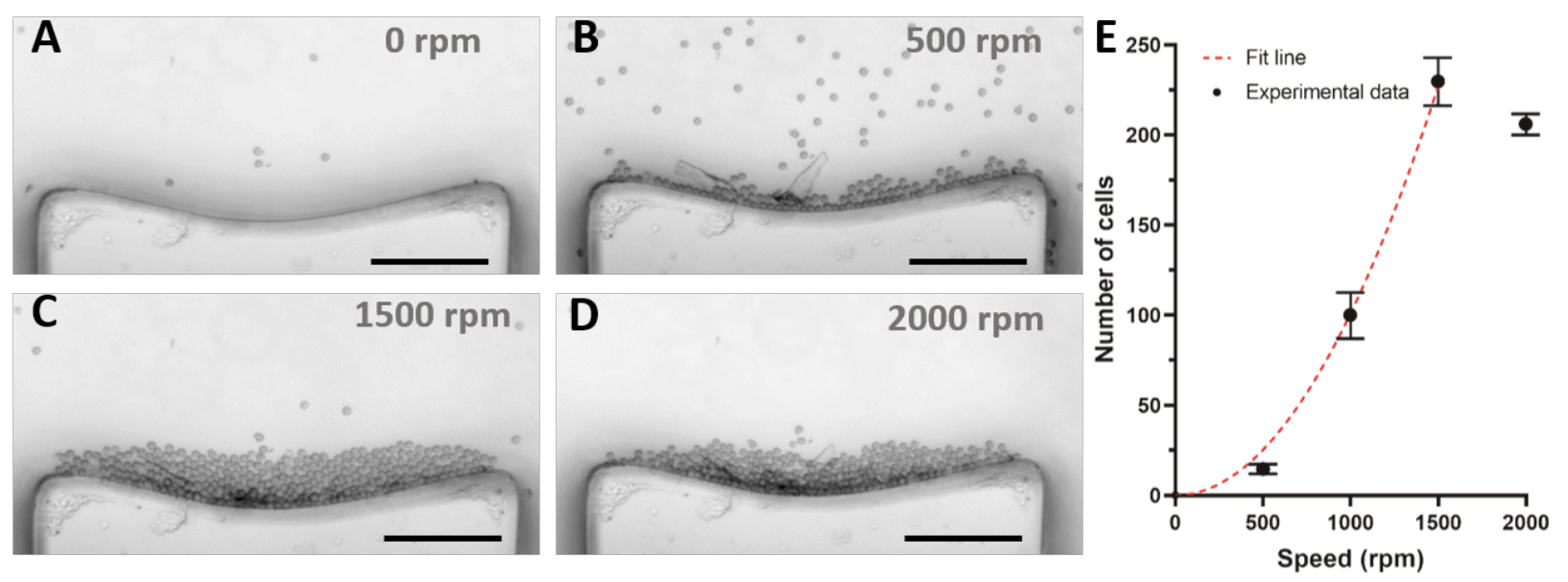

408 Figure 3. Cell alignment via centrifugation. (A-D) Effect of centrifugation speed on the 409 alignment of the cells within the concave barrier. (Scale bar $=100 \mu \mathrm{m})(\mathbf{E})$ Number of aligned 410 cells as a function of rotation speed ( Error bars $=$ mean \pm standard deviation, $n=$ 4115 experimental repeats, $\left.R^{2}>0.98\right)$. 
A

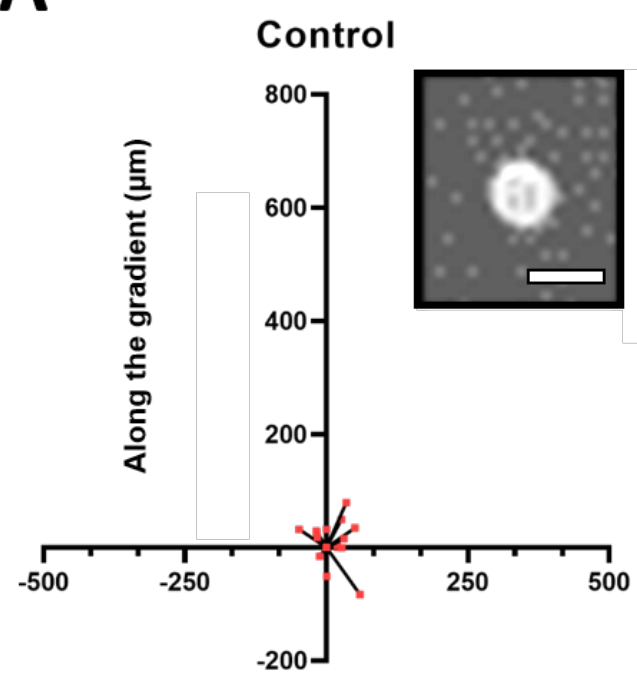

Across the gradient $(\mu \mathrm{m})$
B

100nM fMLP gradient

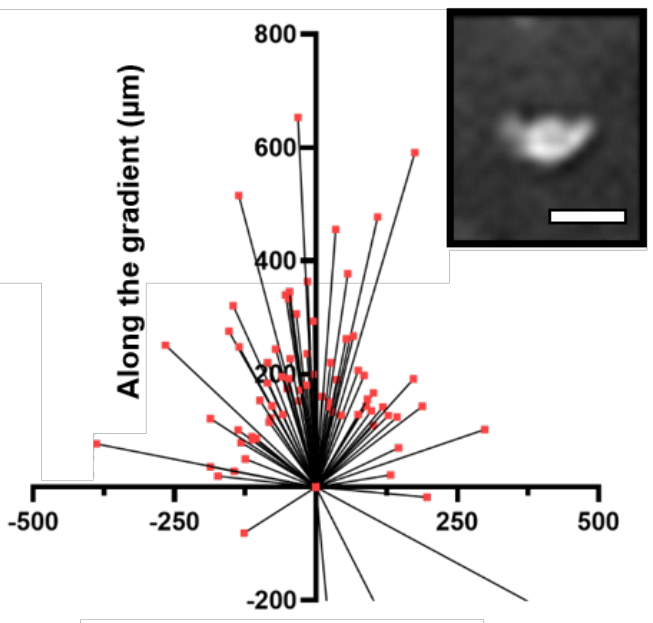

Across the gradient $(\mu \mathrm{m})$

414 Figure 4. Validation of chemotaxis in the microfluidic device. (A) Real-time tracking of the 415 initial and final cell positions after two hours of incubation in the absence of fMLP 416 chemoattractant. Inset: Spherical cell morphology observed under these conditions. (B) Real-time 417 tracking of the initial and final positions after two hours of migration in a $100 \mathrm{nM}$ fMLP gradient. 418 Inset: Elongated morphology adopted by actively migrating cells. (Scale bar $=10 \mu \mathrm{m}$ ) 


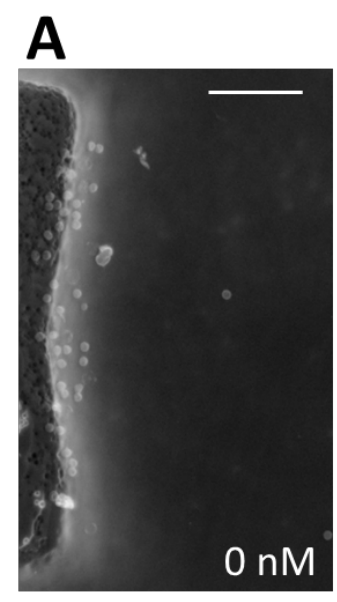

D

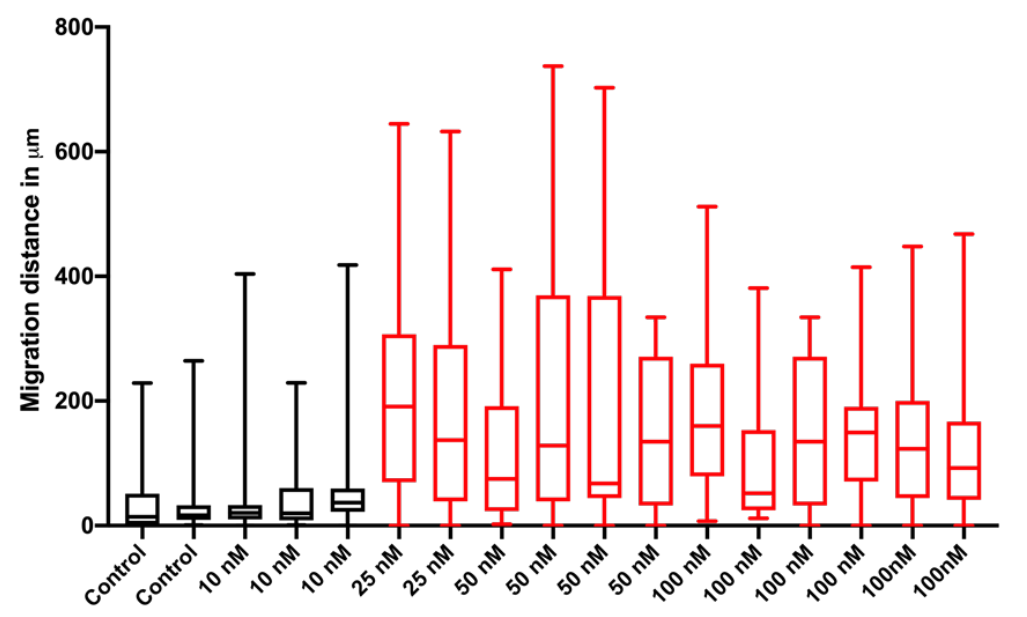

B

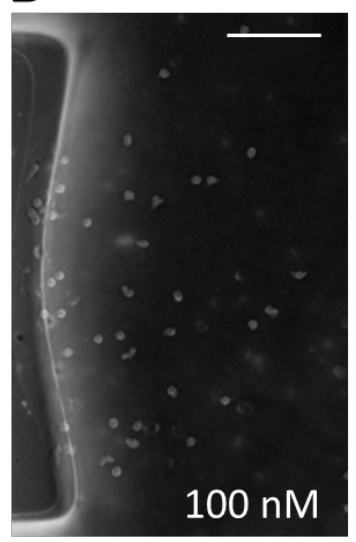

420

421

Figure 5. Endpoint analysis chemotaxis assay. (A-B) Micrographs of cells near the barrier features following alignment and two hours incubation for control (A) and a $100 \mathrm{nM}$ fMLP gradient (B). (Scale bar $=100 \mu \mathrm{m})($ C) Positions of cells along the length of the channel over two hours of migration in a $100 \mathrm{nM}$ fMLP gradient (Error bars indicate standard deviation, $n>240$ ). (D-E) Cell position distribution after chemotaxis as a function of fMLP gradient strength (Error bars indicate standard deviation). Observed differences in the mean of migration distance between $\leqslant 10$ and $\geqslant 25 \mathrm{nM}$ fMLP were statistically significant $(\mathrm{p}<0.005)$. 